Frank A. Chervenak*, Laurence B. McCullough, Amos Grünebaum, Eran Bornstein, Cihat Sen, Milan Stanojevic, Marina Degtyareva and Asim Kurjak

\title{
Professionally responsible advocacy for women and children first during the COVID-19 pandemic: guidance from World Association of Perinatal Medicine and International Academy of Perinatal Medicine
}

https://doi.org/10.1515/jpm-2020-0329

Received July 13, 2020; accepted July 14, 2020; published online August 10, 2020

\begin{abstract}
The goal of perinatal medicine is to provide professionally responsible clinical management of the conditions and diagnoses of pregnant, fetal, and neonatal patients. The New York Declaration of the International Academy of Perinatal Medicine, "Women and children First - or Last?" was directed toward the ethical challenges of perinatal medicine in middle-income and low-income countries. The global COVID-19 pandemic presents common ethical challenges in all countries, independent of their national wealth. In this paper the World Association of Perinatal Medicine provides ethicsbased guidance for professionally responsible advocacy for women and children first during the COVID-19 pandemic. We first present an ethical framework that explains ethical reasoning, clinically relevant ethical principles and professional virtues, and decision making with pregnant patients and parents. We then apply this ethical framework to evidence-based treatment and its improvement, planned home birth, ring-fencing obstetric services, attendance of
\end{abstract}

\footnotetext{
*Corresponding author: Frank A. Chervenak, MD, Department of Obstetrics and Gynecology, Lenox Hill Hospital, 100 East 77th St., New York, NY, 10075, USA, E-mail: fchervenak@northwell.edu Laurence B. McCullough, Amos Grünebaum and Eran Bornstein: Department of Obstetrics and Gynecology, Zucker School of Medicine at Hofstra/Northwell, Hempstead, NY, USA

Cihat Sen: Department of Perinatology, Cerrahpaşa Medical School, University of Istanbul, Istanbul, Turkey

Milan Stanojevic: Department of Neonatology, University Hospital "Sveti Duh", Zagreb, Croatia

Marina Degtyareva: Department of Neonatology, Pirogov Russian National Research Medical University, Moscow, Russia. https:// orcid.org/0000-0002-1769-5430

Asim Kurjak: Department of Obstetrics and Gynecology, University of Zagreb, Zagreb, Croatia; University of Sarajevo, Sarajevo, Bosnia and Herzegovina
}

spouse or partner at birth, and the responsible management of organizational resources. Perinatal physicians should focus on the mission of perinatal medicine to put women and children first and frame-shifting when necessary to put the lives and health of the population of patients served by a hospital first.

Keywords: COVID-19 pandemic; frame-shifting; professional ethics in perinatal medicine; ring-fencing; triage.

\section{Introduction}

The goal of perinatal medicine is to provide professionally responsible clinical management of the conditions and diagnoses of pregnant, fetal, and neonatal patients. Guidance has been provided on the clinical management [1], outcomes [2], and risk factors [3] of COVID-19 during pregnancy. The New York Declaration of the International Academy of Perinatal Medicine, "Women and children First - or Last?" was directed toward the ethical challenges of perinatal medicine in middle-income and low-income countries $[4,5]$. The global COVID-19 pandemic presents common ethical challenges in all countries, independent of their national wealth. The COVID-19 pandemic has become a powerful leveler of differences among countries and peoples. The purpose of this paper is to provide ethics-based guidance for professionally responsible advocacy for women and children first during the COVID-19 pandemic in response to the common ethical challenges that the COVID-19 pandemic presents to perinatal physicians.

\section{Ethical framework}

The ethical framework required for professional ethics in perinatal medicine has two components: ethical reasoning and decision making with patients and parents [6]. 


\section{Ethical reasoning}

Ethical reasoning is not a matter of personal opinion, with one individual's opinion as good as another's. Ethical reasoning is rigorous and therefore more reliably than individual opinion. Ethical reasoning achieves rigor in two steps. The first, ethical analysis requires clarity about the meaning of clinically relevant concepts. The second, ethical argument requires identifying the implications of clear concept for clinical judgment and clinical management based on it.

There are five key concepts in professional ethics in perinatal medicine with clinical implications for the COVID-19 pandemic. These concepts create ethical obligations to act in specified ways. These obligations are limited in that, when obligations are not compatible, ethical reasoning is required to establish priority among them. In the technical language of philosophical ethics, limited ethical obligations are known as prima facie ethical obligations.

The ethical principle of beneficence creates the prima facie ethical obligation to identify and provide clinical management that is predicted in deliberative (evidencebased, rigorous, transparent, and accountable) clinical judgment to result in net clinical benefit for the pregnant, fetal, or neonatal patient. Such clinical management is known as medically reasonable.

The ethical principle of respect for autonomy creates the prima facie ethical obligation to empower the pregnant patient (in obstetrics) or the parents (in pediatrics) to make informed decisions about the clinical management of her condition (pregnancy is a condition not a disease) or diagnoses, or that of her fetus. This process occurs in the following sequence. The physician identifies the medically reasonable forms of clinical management of the patient' or fetus' condition or diagnosis. The physician explains the nature of each of these forms of clinical management, as well as their benefits and significant risks (adverse outcomes that in deliberative clinical judgment should be minimized). The physician should support the patient as she develops her understanding of each of these forms of clinical management and assesses them on the basis of her values and beliefs. With the patient's resulting informed authorization, the physician provides clinical management.

The ethical principle of justice was first articulated in the history of Western philosophy by Aristotle (385-323 $\mathrm{BCE}$ ). Aristotle argued that justice creates the prima facie ethical obligation to treat like cases alike. In healthcare, physicians are trained to group cases by condition or diagnosis. The ethical principle of healthcare justice creates the prima facie ethical obligation to see to it that each pregnant, fetal, and neonatal patient receives medically reasonable clinical management of the patient's condition or diagnosis.

The professional virtue of integrity creates the prima facie ethical obligation to provide clinical management that meets standards of intellectual and moral excellence. Intellectual excellence is achieved by routinely submitting to the discipline of deliberative clinical judgment. Moral excellence is achieved by routinely keeping individual and organizational self-interest systematically secondary.

The professional virtue of self-sacrifice creates the prima facie ethical obligation to take reasonable risks to one's health, life, and other legitimate self-interests in order to fulfill beneficence-based, autonomy-based, and justicebased prima facie ethical obligations to the pregnant, fetal, or neonatal patient. We emphasize that the judgment about reasonable risk be made in an evidence-based process that minimizes bias introduced by the understandable concern to avoid risk to one's health and life. We also emphasize that there is no ethical obligation to take what should be considered, in deliberative clinical judgment, unreasonable risks, e.g., caring for COVID-19 patients without appropriate preventive measures.

\section{Decision making with pregnant patients and with parents}

\section{Decision making with the pregnant patient}

In professional ethics in obstetrics in the perinatal setting, the physician has prima facie ethical obligations to both the pregnant and fetal patient. The physician has beneficence-based and autonomy-based prima facie ethical obligations to the pregnant patient. The physician also has beneficence-based prima facie ethical obligations to the fetal patient. Without exception, the physician should identify all three prima facie ethical obligations. The pregnant patient also has beneficence-based prima facie ethical obligations to the fetal patient. We emphasize that there is no limitless or absolute beneficence-based ethical obligation of the pregnant woman to the fetal patient. This means that she is ethically obligated to take only reasonable risk to herself for fetal benefit from intrapartum management [4].

The informed decision making process should begin with the identification and presentation of the forms of 
clinical management that in deliberative clinical judgment are considered to be medically reasonable, taking into account beneficence-based prima facie ethical obligations to both the pregnant and fetal patient. The pregnant patient should be supported to understand and assess these forms of clinical management. Finally, the physician should be alert to potential controlling influence of others over the pregnant woman's decision-making process, e.g., the mother of a teenage pregnant patient who states that her daughter cannot make decisions for herself when there is no clinical evidence to support this assessment. The physician should prevent such controlling influences from occurring, to ensure that the pregnant patient's decisionmaking process is voluntary and that she remains the ultimate decision maker.

\section{Decision making with parents}

A baby delivered in a hospital becomes a neonatal patient. Clinical management should be guided by professional ethics in pediatrics, which has two components. The first is beneficence-based and is known as the best interests of the child standard. This standard creates the prima facie ethical obligation of the physician to identify and provide medically reasonable clinical management of the neonatal patients' condition or diagnosis. The second is parental permission, a beneficence-based and autonomy-based concept. Parents are also bound by the best interests of the child standard, when their child is a patient. They therefore have a prima facie ethical obligation to authorize medically reasonable clinical management that has been offered or recommended by their child's physician. The parents therefore do not provide consent, because they are not ethically free to refuse such authorization. Parental "permission" is used to signal that their autonomy is limited by the best interests of the child standard [6].

It is important that a pediatrician who is involved in the decision making process with the pregnant woman about intrapartum management think like an obstetrician: the perinatal team has autonomy-based and beneficence-based prima facie ethical obligations to the pregnant patient and beneficence-based prima facie ethical obligations to the fetal patient. The pregnant woman is the ultimate decision maker. It is equally important that the obstetrician who is involved in decision making about the birth plan and subsequent neonatal management think like a pediatrician. The best interests of the child standard should guide the perinatal team in this decision-making process and both parents should provide parental permission.

\section{Clinical applications of the ethical framework \\ Evidence-based treatment and its improvement}

The clinical management of pregnant and neonatal patients with COVID-19 patients should be evidence based, as required by the ethical principles of beneficence and healthcare justice and the professional virtue of integrity. This means that the use of medications or other forms of clinical management should be introduced only in the context properly organized, conducted, and overseen clinical trials. Some physicians - and many patients and their families, as well as public figures - will claim that desperate cases justify desperate measures. Perinatal physicians should reject this approach in favor of its professionally responsible alternative: desperate cases justify deliberative measures. These can include compassionate use of investigational clinical management outside clinical trials but only if such use does not result in denial of access for research subjects in an approved trial, its use is reviewed and approved by the research oversight body with jurisdiction (Institutional Review Board in the United States and Research Ethics Committee in other countries), and the informed consent process makes it unambiguously clear that the clinical management is experimental (its outcome cannot be reliably predicted) [6].

\section{Planned home birth}

Since the onset of the COVID-19 pandemic more pregnant women may indicate that they are considering planned home birth, as a way to prevent infection that could occur in the hospital setting [7]. However, by following accepted infection control measures in the hospital, the risk of infection to the pregnant woman and her newborn is considered negligible. "Negligible" means that the risk is so small that planning for delivery in the hospital should not be altered. The same is the case for newborns. This clinical reality should be explained to the pregnant woman. Respect for autonomy, as explained above, creates the prima facie ethical obligation to support her in developing her understanding that the infection risk in the hospital is negligible and her assessment of it. In fact, infections as a cause of neonatal deaths are more likely at planned home births than at hospital births [8]. In some countries such as the United States home birth is not well integrated into the health system, and there are documented risks of increased neonatal 
morbidity and mortality [9]. In this context, the obstetrician should recommend against planned home birth and recommend planned hospital birth. In the few countries with well-integrated home birth, the woman's informed decision for planned home birth may not be unreasonable.

\section{Ring-fencing obstetric services}

The phrase "ring-fencing" refers to an ancient practice in the British Isles of creating rings or fences of stone to prevent livestock from escaping and entering. It has become to be used in finance science to indicate the use of management techniques to protect financial assets from inappropriate encroachment and misuse [10]. The phrase applies in the hospital setting to protect resources required for essential patient care from being diverted to other hospital services.

The need for ring-fencing obstetric services has arisen during the COVID-19 pandemic In the United States, when some government officials have declared termination of pregnancy an "elective" procedure that can be prohibited, to conserve hospital resources for COVID-19 patients. Ringfencing is ethically justified to protect essential patient services from being unjustifiably limited by government officials.

These examples raise the question of when ring-fencing of obstetric services should be considered ethically justified, i.e., whether diversion of resources is compatible with beneficence-based, justice-based, and integrity-based ethical obligations to obstetric patients. The ethical principle of healthcare justice creates the prima facie ethical obligation to provide every patient with medically reasonable clinical management. Failure to do so results in substandard or no clinical management, which is ruled out by the principle of beneficence and the professional virtue of integrity. Medically reasonable obstetric care includes termination of pregnancy, rapid access to cesarean delivery, and the capacity to rapidly respond to major maternal complications such a hemorrhage. Medical reasonable obstetric care is essential obstetric care and therefore should never be labeled "elective" as a way to relegate it to secondary priority in a healthcare organization.

\section{Attendance of spouse or partner at birth}

Medically reasonable clinical management has biological, as well as psychological and social, components. The psychiatrist George Engel (1913-1999) introduced the biopsychosocial concept of health and disease to emphasize the complex nature of health and disease [11]. He also aimed to prevent the scientific and clinical errors that can occur when the physician focuses only on the biomedical component of health and disease. This is called biomedical reductionism. For example, a woman who is single mother of two small children at home may be reluctant to authorize cesarean delivery because she in uncertain about whether she can arrange for the additional days of childcare while she recovers from surgery. Biomedical reductionism could blind the obstetrician to this patient's reasoning and lead to unacceptable delay of cesarean delivery.

Attendance by the pregnant woman's support person, e.g., spouse or partner, is an essential component of medically reasonable obstetric care. In the early days of the COVID-19 pandemic in New York City some obstetric services barred support persons from labor and delivery. The Governor of New York State intervened to override these policies [10]. Barring attendance was not required for infection control when the support person was willing to comply with all infection control measures. The result was that the risk of psychosocial harm, including possible longterm psychosocial harm regarding family formation, was increased with no offsetting benefit. The ethical principle of beneficence and the professional virtue of integrity rule out this line of mistaken reasoning. The take-home lesson is that the understandable stress and anxiety of a pandemic that causes a surge of admission of very sick patients can short-circuit evidence-based ethical analyzis. One of the most important behavioral changes that professional ethics promotes is taking the time to think through stressful ethical challenges, by analogy to taking a time out before a surgical procedure commences.

\section{The responsible management of limited organizational resources}

\section{The necessity of frame-shifting}

A common feature of pandemics is that the number of patients and their clinical needs can overwhelm the resources of healthcare organizations. When this occurs, it may necessary to triage space, medications, and lifesustaining treatment, in ascending order of clinical impact. Triage is not about meeting the needs of an individual patient, the one-at-a-time clinical application of the concept of medically reasonable. Instead, the concept of medically reasonable is applied to the population of patients served by a healthcare organization. This requires "frame-shifting" [12]. The goal in an overwhelming pandemic is to reduce mortality as much as possible in this population of patients and, to the extent that resources allow, minimize morbidity and disability among survivors. 
This may mean that some patients will be allowed to die or be undertreated. At the clinical level, this is ruled out by the ethical principle of beneficence; at the population level this is ruled in by the ethical principle of healthcare justice.

This is not easy change to make for any physician. We have used the word "frame-shifting" to signal how jarring and unwelcome this change is. This has important implication for triage of life-sustaining treatment, which we address below.

\section{Allocation of space}

In order to continue to provide access to a hospital by COVID-19 and other patients requiring hospitalization, hospitals have repurposed space to ensure access for COVID-19 patients who require admission, to maintain access to the emergency department [12]. A number of measures have been taken. An early step was to eliminate elective surgery. Admission from the emergency department into a hospital bed had to be expedited, so that patients in ambulances could be admitted to the emergency department without clinically unacceptable delay while waiting in the ambulance. It was also crucial to free up ambulances so that they could go to their next urgent calls without clinically unacceptable delay. To meet the higher level of care required by the most seriously ill COVID-19 patients, hospitals have had to add critical care beds in addition to existing critical care units, in effect creating new critical care units in such spaces as operating rooms and pre-operative and post-operative areas. The goal of these and other measures has been to prevent triage of lifesustaining treatment, which is addressed below.

In order to free up even more hospital space, some departments of obstetrics and gynecology have been asked to plan for relocation to a new site, removed from the hospital [10]. The ethical principal of healthcare justice should guide leaders in obstetrics in response to such requests. This ethical principle creates a prima facie ethical obligation to protect the space required to provide each patient with medically reasonable clinical management. Such clinical management includes rapid access to operating rooms for cesarean deliveries. The "time from decision to incision" requires that access be in contiguous space. Medically reasonable obstetric management also includes the involvement of consultants from other specialties, to ensure timely and effective response to sudden intrapartum complications. Finally, medically reasonable clinical management includes rapid and effective access to critical care services when a pregnant or gynecologic patient has a life-threatening condition. In these and other clinical circumstances the location of obstetrics and gynecology in the hospital is required by the healthcare-justice-based ethical obligation to ensure that every patient receives medically reasonable clinical management of her condition or diagnosis [12].

Extreme circumstances may arise in which the hospital needs to maximize space available to provide lifesustaining treatment to all eligible patients, to prevent having to ration such treatment. Ring-fencing obstetric and gynecologic services may prevent the hospital from achieving this goal. From the population-based perspective, the outcome of failing to prevent rationing would be incompatible with the prima facie ethical obligation of the hospital under the ethical principle of healthcare justice. Frame-shifting is required so that an unbiased plan for relocating obstetric and gynecologic services can be evaluated for its compatibility with the ethical principle of healthcare justice. Failure to surge the capacity for lifesustaining treatment can reliably be predicted to result in more mortality and major morbidity from COVID-19 than the increased risk of mortality and morbidity from relocating obstetric and gynecologic services out of the hospital. There is, however, a healthcare-justice-based imperative: the out-of-hospital space should be made as safe as possible by meeting the conditions described above.

\section{Allocation of medications}

DeJong, Chen, Lo have recently provided ethics-based guidance on responsibly managing scarce medications during the COVID-19 pandemic [13]. Frame-shifting to a population-based perspective, they prioritize reducing mortality as much as possible and emphasize that patients' preferences will sometimes justifiably be limited in order to achieve this priority. On this basis they make six recommendations that we support as the basis for creating professionally responsible organizational policy.

(1) Allocation should be evidence-based.

(2) Prioritization should not exclude patients based on age, disability, religion, race or ethnicity, national origin gender, sexual orientation, or perceived quality of life.

(3) Patients already receiving appropriate medications for other serious illness should continue to receive their medication.

(4) Clinical judgment about which patients might most benefit should be based on the best available evidence.

(5) In the eligible population that results from applying the first four recommendations, to be fair allocation should be based on randomization. It would be ethically permissible to prioritize essential healthcare professionals before randomization is applied. 
(6) Physicians who are tasked to explain to a patient or patient's family that the patient will not receive the scarce medication should receive full organizational back-up and support [12].

\section{Allocation of life-sustaining treatment}

Every effort should be made to maximize the hospital's capacity for life-sustaining treatment, utilizing appropriate space and personnel, to prevent the need for triage. Frameshifting to a population-based perspective will be very challenging, because the triage officer will be deciding which patients will be provided access to life-sustaining treatment and which will. Organizational leaders should designate physicians who are held in the highest professional regard to serve as triage officers. These physicians should have no direct responsibility for patient care, to prevent conflicts of commitment. The sole criterion should be maximal reduction of the risk mortality at discharge with at least some interactive capacity among survivors in the patient population, using evidence-based standards [14]. The triage officers should use scoring systems that include accepted and clinically applicable measures as the basis for predicted mortality and accepted and clinically applicable measures for predicted absence of interactive capacity. No other clinical considerations should be allowed, such predicted post-discharge quality of life or disability, because such clinical considerations introduce the very biases that DeJong, Chen, and Lo correctly exclude [13].

We have deliberately used the plural, triage "officers." We have done so out of recognition that the role of triage will be extremely demanding and almost take a psychological toll on the physicians assigned this momentous professional responsibility. Rotating the responsibility should be seen as an essential safeguard for the integrity of the triage process. If time allows, an additional safeguard is periodic retrospective review to prevent drift away the focus on maximal reduction of the risk mortality at discharge with at least some interactive capacity among survivors in the patient population.

\section{Conclusions}

The COVID-19 pandemic has had systemic and sometimes devastating impact on public and national economies in many countries. The medical community has adapted rapidly and developed approaches to manage the scientific and clinical aspects of the pandemic in a professionally responsible way. The purpose of this paper has been to equip perinatal physicians will an ethically justified approach so that they can accomplish two vital tasks: Focusing without hesitation on the mission of perinatal medicine to put women and children first and frame-shifting when necessary to put the lives and health of the population of patients served by a hospital first.

Research funding: None declared.

Author contributions: All authors have accepted responsibility for the entire content of this manuscript and approved its submission.

Competing interests: Authors state no conflict of interest.

\section{References}

1. Api O, Sen C, Debska M, Saccone G, D'Antonio F, Volpe N, et al. Clinical management of coronavirus disease 2019 (COVID-19) in pregnancy: recommendations of WAPM-world association of perinatal medicine. Perinat Med, https://doi. org/10.1515/jpm-2020-0265 [published online ahead of print 2020 Jul 21].

2. Di Mascio D, Khalil A, Saccone G, Rizzo G, Buca D, Liberati M. Outcome of Coronavirus spectrum infections (SARS, MERS, COVID 1-19) during pregnancy: a systematic review and meta-analysis [published online ahead of print, 2020 Mar 25]. Am J Obstet Gynecol MFM, 2020;2:100107.

3. Boelig RC, Manuck T, Oliver EA, Di Mascio D, Saccone G, Bellussi $\mathrm{F}$, et al. Labor and delivery guidance for COVID-19. Am J Obstet Gynecol MFM. 2020;2:100110.

4. Chervenak FA, McCullough LB. International academy of perinatal medicine. Women and children first-or last? The New York declaration. Am J Obstet Gynecol 2009;201:335.

5. Chervenak FA, McCullough LB. Women and children first: transforming a historic defining moment into a contemporary ethical imperative. Am J Obstet Gynecol 2009;201:e351-515.

6. McCullough LB, Coverdale JH, Chervenak FA. Professional ethics in obstetrics and gynecology. New York, Cambridge: Cambridge University Press; 2020.

7. Grünebaum A, McCullough LB, Bornstein E, Klein R, Dudenhausen JW, Chervenak FA. Professionally responsible counseling about birth location during the COVID-19 pandemic. J Perinat Med 2020; 48:450-2.

8. Grünebaum A, McCullough LB, Arabin B, Dudenhausen J, Orosz B, Chervenak FA. Underlying causes of neonatal deaths in term singleton pregnancies: home births vs. hospital births in the United States. J Perinat Med 2017;45:349-57.

9. Grünebaum A, McCullough LB, Orosz B, Chervenak FA. Neonatal mortality in the United States is related to location of birth (hospital vs. home) rather than the type of birth attendant. Am J Obstet Gynecol 2020;S0002-9378:30067-3. [published online ahead of print, 2020 Feb 7].

10. Grünebaum A, Dudenhausen J, McCullough LB, Chervenak FA. Women and children first: the need for ringfencing during the COVID-19 pandemic. J Perinat Med 2020;48:305-6.

11. Engel GL. The need for a new medical model: a challenge for biomedicine. Science 1977;196:129-36. 
12. Chervenak FA, Grünebaum A, Bornstein E, Wasden S, Katz A, Rochelson BL, et al. Expanding the concept of the professional integrity of obstetrics during a public health emergency. J Perinat Med 2020;48:435-7.

13. Dejong $C$, Chen AH, Lo B. An ethical framework for allocating scarce inpatient medications for COVID-19 in the US. JAMA
2020. https://doi.org/10.1001/jama.2020.8914 [published online ahead of print, 2020 May 15].

14. McCullough LB. In response to COVID-19 pandemic physicians already know what to do. Am J Bioeth 2020:1-4. https://doi.org/ 10.1080/15265161.2020.1754100 [published online ahead of print, 2020 Apr 23]. 